\title{
Comorbid obstructive sleep apnoea and chronic obstructive pulmonary disease and the risk of cardiovascular disease
}

\author{
Walter T. McNicholas ${ }^{1,2}$ \\ ${ }^{1}$ First Affiliated Hospital of Guangzhou Medical University, Guangzhou 510000, China; ${ }^{2}$ School of Medicine, University College Dublin, Dublin, \\ Ireland \\ Correspondence to: Prof. Walter McNicholas, MD. Department of Respiratory and Sleep Medicine, St. Vincent's University Hospital, Elm Park, \\ Dublin 4, Ireland. Email: walter.mcnicholas@ucd.ie.
}

\begin{abstract}
Chronic obstructive pulmonary disease (COPD) and obstructive sleep apnoea (OSA) syndrome are both highly prevalent, affecting at least $10 \%$ of the general adult population, and each has been independently associated with an increased risk of cardiovascular disease. The presence of both disorders together, commonly referred to as the overlap syndrome, is also highly prevalent, although various clinical and pathophysiological factors associated with COPD may increase or decrease the likelihood of OSA. Lung hyperinflation reduces the likelihood of obstructive apnoea, whereas right heart failure increases the likelihood as a result of rostral fluid shift causing upper airway narrowing in the supine position while asleep. Furthermore, upper airway inflammation associated with OSA may aggravate lower airway inflammation in COPD. The proposed mechanisms of cardiovascular disease in each disorder are similar and include systemic inflammation, oxidative stress, and sympathetic excitation. Thus, one could expect that the prevalence of co-morbid cardiovascular disease would be higher in the overlap syndrome but, with the exception of pulmonary hypertension, there are few published reports that have explored this aspect in depth. Hypoxia is more pronounced in patients with the overlap syndrome, especially during sleep, which is likely to be the principal factor accounting for the recognised higher prevalence of pulmonary hypertension in these patients. Cardiac sympathetic activity is increased in patients with the overlap syndrome when compared to each disorder alone, but echocardiographic evidence of left ventricular strain is no greater in overlap patients when compared to COPD alone. While survival might be expected to be worse in overlap patients, recent evidence surprisingly indicates that the incremental contribution of lung function to mortality diminishes with increasing severity of OSA. Identification of co-morbid OSA in patients with COPD has practical clinical significance as appropriate positive airway pressure therapy in COPD patients with co-existing OSA is associated with improved morbidity and mortality.
\end{abstract}

Keywords: Obstructive sleep apnoea (OSA); chronic obstructive pulmonary disease (COPD); overlap syndrome; cardiovascular disease; continuous positive airway pressure (CPAP)

Submitted Jun 27, 2018. Accepted for publication Oct 25, 2018.

doi: $10.21037 /$ jtd.2018.10.117

View this article at: http://dx.doi.org/10.21037/jtd.2018.10.117

\section{Introduction}

Chronic obstructive pulmonary disease (COPD) and obstructive sleep apnoea (OSA) syndrome are each highly prevalent with recent population-based studies indicating that about $10 \%$ of the general adult population have one or other disorder $(1,2)$. However, the prevalence of isolated sleep disordered breathing (SDB) as measured by the frequency of apnoeas or hypopnoeas per hour of sleep [apnoea-hypopnoea index (AHI)] without accompanying symptoms such as sleepiness is considerably higher with recent studies indicating that up to $50 \%$ of adult males have significantly elevated AHI (3), and even higher numbers in the elderly. Based on these prevalence figures, up to half of 


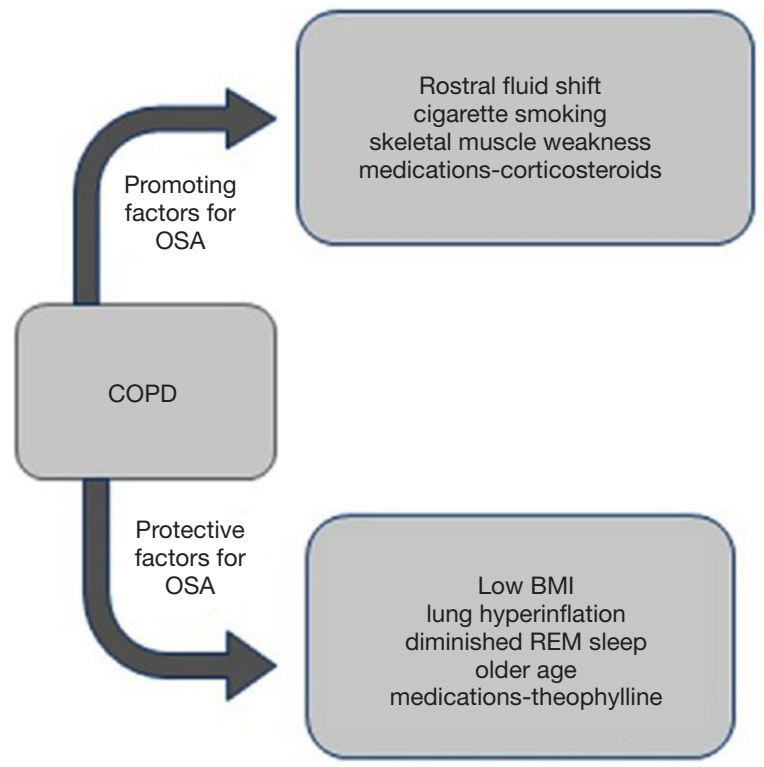

Figure 1 Interactions between COPD and OSA that may influence the prevalence of the overlap syndrome [reproduced from (9)]. COPD, chronic obstructive pulmonary disease; OSA, obstructive sleep apnoea; BMI, body mass index; REM, rapid-eye-movement.

patients with COPD will likely have at least some degree of SDB based on chance association alone. Furthermore, COPD alone is associated with sleep disturbance (4), which can result in significant negative effects on quality of life (5).

COPD and OSA are each independently associated with an increased prevalence of cardiovascular disease $(6,7)$ and there are considerable overlaps in pathophysiological mechanisms which include systemic inflammation, oxidative stress, and sympathetic excitation (8). Thus, one could predict that patients with both COPD and OSA, referred to as overlap syndrome, may have a greater risk of cardiovascular co-morbidity than with either disorder alone, although specific evidence is limited in this regard. The present review discusses the epidemiology of the overlap syndrome and its association with cardiovascular disease, and reviews the evidence regarding mechanisms and outcomes of this association.

\section{Epidemiology of overlap syndrome}

While the overlap syndrome should be highly prevalent based on chance association, there are many features of COPD that influence the likelihood of co-existing OSA (9), which are summarised in Figure 1. Factors in COPD that increase the likelihood of OSA include rostral fluid shift while in the supine position at night (10), which is particularly likely in patients with peripheral oedema due to right heart failure that is present in a minority of patients with COPD. Additional factors include upper airway oedema and inflammation due to cigarette smoking and upper airway muscle weakness that may be aggravated by corticosteroid therapy, both inhaled and oral (11). Other COPD-related factors protect against the development of OSA including low body mass index (BMI) and lung hyperinflation, which is associated with reduced upper airway collapsibility (12) and a lower AHI (13). Additional potentially protective factors include older age (14), medications such as theophylline (15), and also the diminished amounts of rapid-eye-movement (REM) sleep that are common in patients with COPD (4). In general terms, factors promoting OSA are more associated with the predominant chronic bronchitis phenotype (16) whereas factors protective against OSA are more associated with the predominant emphysema phenotype (13). Factors relating to OSA that may contribute to COPD include upper airway inflammation (17) and chronic intermittent hypoxia, which has been reported to increase lung inflammation and damage (18).

Several reports have examined the prevalence of overlap syndrome in population studies of patients with COPD (19-21) and separately in population studies of patients with OSA $(14,22)$. These reports have indicated widely different prevalence figures for OSA in patients with COPD and for COPD in patients with OSA with reported prevalence figures ranging from $7.6 \%$ to $55.7 \%$ for COPD in patient populations with OSA and from $2.9 \%$ to $66 \%$ for OSA in patient populations with COPD (23). The widely differing prevalence figures likely reflect a wide variety of factors that include differences in patient populations and clinical phenotypes, sleep study recording techniques, in addition to different definitions of SDB and OSA. Earlier respiratory recordings such as thermistor were less sensitive, and likely gave a lower AHI than later recordings such as those based on nasal pressure. Furthermore, none of the published reports of OSA prevalence in COPD populations have characterized patients based on clinical phenotype, which is likely to have a major impact on the predisposition to OSA based on the characteristics indicated in Figure 1. Factors that have been found to increase the likelihood of OSA in COPD include higher BMI and continued smoking (20), both representing risk factors for cardiovascular disease. Only two general population studies of overlap syndrome that included formal sleep studies have been reported which gave prevalence figures of one and 2.7 percent $(21,24)$. 


\section{Cardiovascular disease in the overlap syndrome}

Both COPD and OSA are associated with increased risk of cardiovascular disease. A recent meta-analysis reported a significantly higher prevalence of cardiovascular comorbidities in COPD patients (OR 1.90, 95\% CI: $1.59-2.28 ; \mathrm{P}<0.00001)$ based on data from 11 studies that involved over 45 million COPD patients and a similar number of control subjects (25), which is similar to an earlier systematic review that found an odds ratio (OR) of 2.46; 95\% CI: 2.02-3.00; $\mathrm{P}<0.0001$ (7). Cardiac dysfunction is particularly likely during exacerbations of COPD (26), which may be predicted by elevated levels of BNP, NTproBNP, and/or troponins (27). Cardiovascular disease is also more prevalent in OSA with a relative risk of 2.48 (1.98-3.10) for total cardiovascular disease (CVD), 1.37 $(0.95-1.98)$ for coronary heart disease (CHD), and 2.02 (1.40-2.90) for stroke in one recent meta-analysis (28). OSA particularly predisposes to hypertension and cardiac dysrhythmias, especially atrial fibrillation (6), but has also been associated with other cardiovascular disorders including coronary artery disease and congestive heart failure (6). Based on these recognised risks for various cardiovascular diseases associated with COPD and OSA alone, one should expect a greater risk for cardiovascular disease in patients with the overlap syndrome. However, epidemiological data are limited on this aspect although a recent retrospective study reported a higher prevalence of hypertension, diabetes and the metabolic syndrome in patients with the overlap syndrome compared to OSA alone (29).

Pulmonary hypertension has long been recognised as more prevalent in patients with the overlap syndrome when compared to either disorder alone (30). This higher prevalence is likely a consequence of the greater degree of hypoxaemia in patients with the overlap syndrome (31), especially during sleep. Furthermore, Sharma and coauthors reported in a small cohort of 18 COPD patients, 7 of whom had co-existing OSA, that overlap patients had a higher right ventricular mass index on cardiac magnetic resonance imaging (32), which is likely a consequence of pulmonary hypertension. Atrial fibrillation is a recognised complication of OSA, but patients with overlap syndrome have been reported to have a greater risk of new onset atrial fibrillation when compared to patients with OSA alone (33). Coronary artery disease is also commonly associated with both COPD and OSA but there are no reported data comparing the incidence of coronary disease in overlap patients with those having COPD or OSA alone. Although the epidemiological evidence is limited for increased cardiovascular disease in the overlap syndrome, the importance of recognising co-existing OSA in patients with COPD is underlined by the interesting recent report of Castro-Grattoni and co-authors demonstrating in a rodent model that cardiovascular changes induced by chronic intermittent hypoxia can be reversed by normoxia (34).

\section{Mechanisms of cardiovascular disease in the overlap syndrome}

The mechanisms of cardiovascular disease in COPD and OSA are complex and not fully understood (8). In COPD, mechanisms include hypoxia, systemic inflammation and oxidative stress, in addition to sympathetic excitation and vascular dysfunction (35). Vascular dysfunction in COPD is likely a result of several factors including inflammation, endothelial dysfunction, and connective tissue degradation, the latter resulting from increased elastolytic activity and collagen deposition. In OSA, mechanisms also include hypoxia, inflammation and oxidative stress, in addition to sympathetic excitation, endothelial dysfunction, and metabolic dysregulation $(6,36)$. The pattern of hypoxia in OSA is intermittent, which especially predisposes to inflammation and oxidative stress (37). Indeed, there are important differences in the pattern of nocturnal hypoxaemia between COPD, OSA, and the overlap syndrome (38). In COPD, nocturnal hypoxaemia is typically modest, with intermittent hypoxaemia particularly evident in REM sleep. In OSA, hypoxaemia is typically intermittent, with periods of oxygen desaturation during apnoea/ hypopnoea interspersed with resaturation to relatively normal levels in between episodes of SDB, unless there is some associated hypoventialtion. In contrast, patients with the overlap syndrome also show intermittent hypoxaemia but typically remain hypoxaemic between episodes of SDB, thus demonstrating intermittent desaturation from a baseline hypoxaemia (Figure 2). These different profiles of oxygenation have important implications for the molecular responses to hypoxaemia in these disorders. In particular, intermittent hypoxaemia preferentially activates the pro-inflammatory transcription factor nuclear factor kappa-B $(\mathrm{NF}-\kappa \mathrm{B})$ and its downstream products including tumor necrosis factor alpha (TNF- $\alpha)(39,40)$. The baseline sustained hypoxemia that develops in patients with the overlap syndrome predisposes to other molecular responses relevant to the mechanisms of cardiovascular 

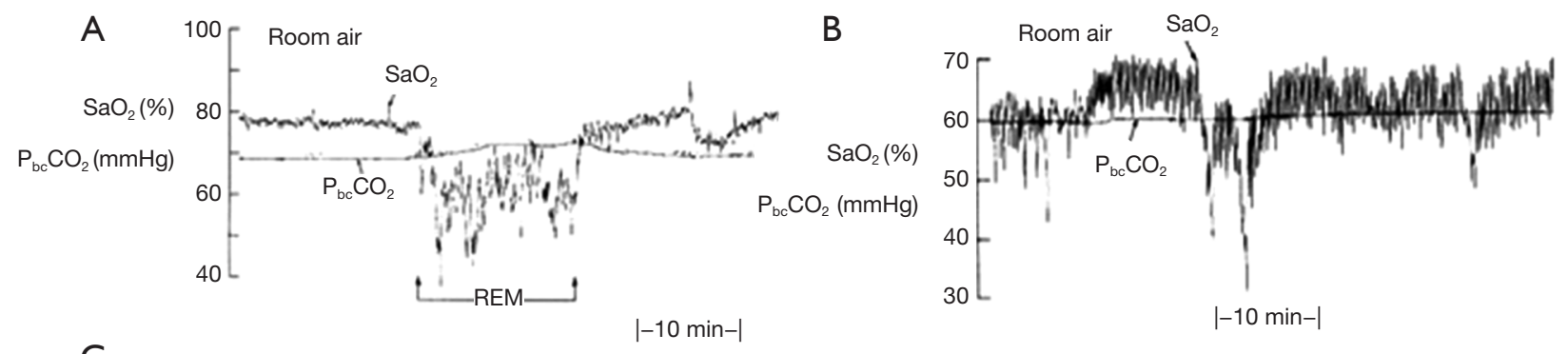

C

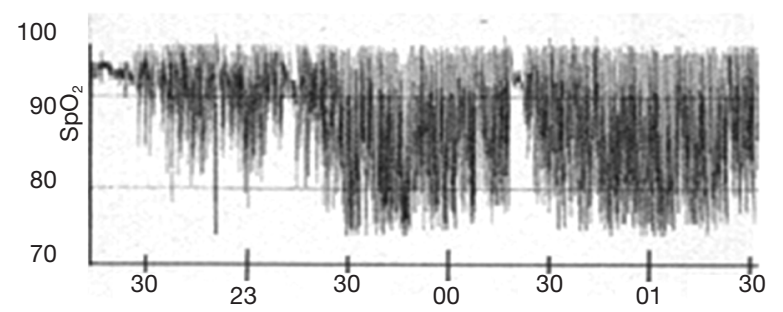

Figure 2 Different patterns of oxygen desaturation during sleep in patients with (A) COPD, (B) overlap syndrome and (C) OSA. COPD, chronic obstructive pulmonary disease; OSA, obstructive sleep apnoea; $\mathrm{SaO}_{2} / \mathrm{SpO}_{2}$, oxygen saturation; $\mathrm{PtcCO}$, transcutaneous carbon dioxide tension [adapted from (38)].

disease, especially via activation of the transcription factor pathway mediated by hypoxia inducible factor- 1 alpha $(\mathrm{HIF}-1 \alpha)$ (39) and related downstream products such as vascular endothelial growth factor (VEGF). Furthermore, there is evidence of interaction between the NF-kB and HIF-1 pathways in the transcriptional responses to hypoxia (41). Sympathetic excitation is common in COPD and contributes to exercise limitation (42), and is also common in OSA where it represents a key factor in the pathogenesis of nocturnal elevation of blood pressure (43).

Thus, it is evident that there are considerable overlaps in the mechanisms of cardiovascular disease in COPD and OSA, although different factors may predominate in each disorder. For example, obesity is an important mechanism of inflammation in OSA (44), whereas skeletal muscle wasting is a source of inflammation in COPD (45). Potential overlapping mechanisms of cardiovascular disease in COPD and OSA are schematically illustrated in Figure 3. The shared mechanisms of cardiovascular disease in COPD and OSA should make it likely that these mechanisms would be enhanced in patients with the overlap syndrome. However, there are little published data on this aspect. One report compared a small population of 14 patients with the overlap syndrome with patients having COPD or OSA alone, and assessed cardiac sympathetic activity by heart rate variability, which is a valuable tool to investigate the sympathetic and parasympathetic components of the cardiac autonomic

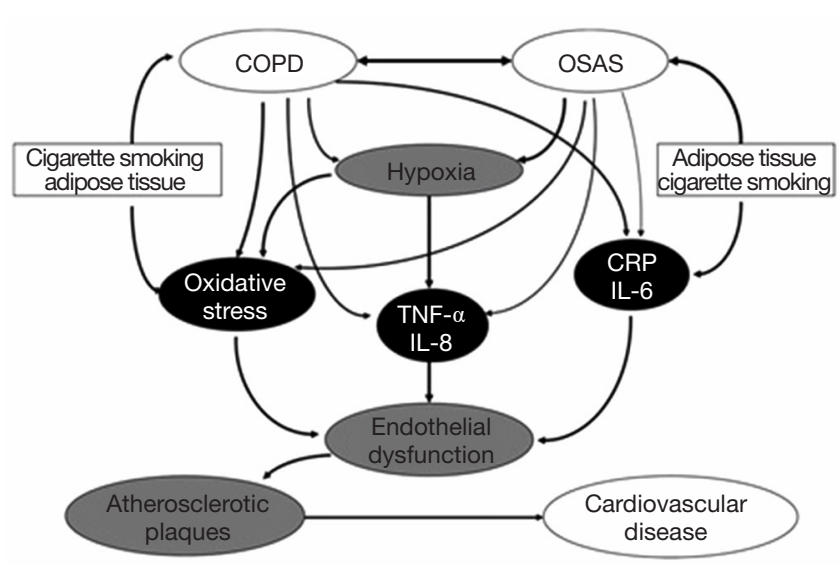

Figure 3 Interactions of COPD and OSAS in inflammatory mechanisms that predispose to cardiovascular disease. Both COPD and OSAS are associated with elevated levels of CRP and IL-6, in addition to TNF- $\alpha$ and IL-8, and are also associated with oxidative stress. However, cigarette smoking and obesity are confounding variables in these associations. Hypoxia is a key factor in elevated TNF- $\alpha$ production in OSAS, which is particularly relevant to the overlap syndrome. In OSA, and to a lesser extent COPD, the hypoxia is usually intermittent, which is particularly pro-inflammatory. Each inflammatory pathway has been associated with atherogenesis and subsequent cardiovascular disease. COPD, chronic obstructive pulmonary disease; OSA, obstructive sleep apnoea; CRP, c-reactive protein; IL-6, interleukin-6; TNF- $\alpha$, tumor necrosis factor alpha; IL-8, interleukin-8. Reproduced from (8). 
nervous system (46). The authors found that overlap patients had significantly higher low frequency to high frequency ratio, indicating higher sympathetic modulation of heart rate, as compared with patients with OSA or COPD alone. Furthermore, the ratio correlated with both AHI and lung residual volume. Another report also indicated that subjects with COPD-OSA overlap present marked sympathetic modulation compared to those with COPD alone and the presence of overlap has a negative impact on functional capacity regardless of the severity of lung disease (47). A further report found evidence of left ventricular dysfunction in patients with COPD that was not significantly greater in the presence of co-existing OSA (48). Sleep itself may have adverse cardiovascular effects in COPD as indicated in the report of Grote and co-authors demonstrating that REM sleep imposes a vascular load on patients with COPD that is independent of OSA (49).

\section{Management and outcomes}

The management of patients with the overlap of COPD and OSA should take into account optimum management principles for each separate disorder, and should particularly reflect the balance of the two disorders in the overall clinical presentation of each patient. Thus, appropriate management of the COPD component should include appropriate bronchodilator therapy, typically inhaled, which in the context of the overlap syndrome may have the added benefit of improving nocturnal oxygen saturation levels $(50,51)$. Theophylline, which may benefit both COPD and OSA $(15,52)$, may not be appropriate if there is co-morbid cardiac disease because of the recognised risk of cardiac side effects, and is not commonly used nowadays in clinical practice. Supplemental oxygen therapy may be appropriate in the presence of significant hypoxaemia (53), which is typically more pronounced at night, and will likely benefit co-morbid pulmonary hypertension $(31,54)$.

Non-invasive pressure support is a key aspect in the management of patients with the overlap syndrome and choice of the most appropriate pressure modality should reflect the balance of the two disorders in the individual patient presentation. Where OSA predominates, standard continuous positive airway pressure (CPAP) is the most appropriate option, either in the fixed or autoadjusting pressure mode. Where the COPD element is prominent, and particularly if there is evidence of associated hypercapnic chronic respiratory failure, noninvasive ventilation (NIV) in the form of bi-level positive airway pressure (BPAP) may be more appropriate by providing an additional element of assisted ventilation. The benefit of BPAP in patients with chronic respiratory failure due to COPD is not fully accepted (55), although does appear to improve outcomes when set to pressures sufficient to markedly reduce carbon dioxide levels (56). Furthermore, a recent report indicates that the addition of home noninvasive ventilation to oxygen supplementation significantly prolonged time to readmission or death in patients hospitalised with an exacerbation of COPD associated with hypercapnia (57).

The benefits of CPAP are more clearly established in patients with the overlap syndrome. Marin and co-authors reported improved long-term survival and lower rate of hospitalizations in over 200 overlap patients treated with CPAP compared to those not treated, and the outcomes of those overlap patients treated with CPAP was similar to patients with COPD alone over a median follow-up period of 9.4 years (Figure 4) (58). Cardiovascular disease accounted for $28 \%$ of deaths. Similar findings were also reported by Machado and co-authors in 95 overlap patients followed for 10 years (59) and the report of Stanchina and co-authors indicated that hours of CPAP use and age were independent predictors of mortality (HR 0.71 and 1.14, $\mathrm{P}<0.001,0.002)$. These findings emphasise the importance of identifying co-morbid OSA in COPD patients, as not doing so with resulting failure to initiate appropriate pressure support may result in increased morbidity and mortality. However, the above studies are observational in nature and prospective randomised trials are required to address outcomes of therapy in overlap patients.

While one could anticipate that the interaction of COPD and OSA in the individual patient would adversely impact survival, the evidence in this regard is not clearcut. In particular, the recent report of Putcha and coauthors (60), based on data from 6173 subjects in the Sleep Heart Health Study having home polysomnography (PSG), indicated that all-cause mortality was higher in those with SDB (AHI $\geq 5$ events/h) compared to those without SDB but, paradoxically, decreasing lung function was associated with increasing mortality to a greater extent in subjects without SDB compared to those with SDB. Furthermore, the incremental influence of lung function on all-cause mortality was less with increasing severity of SDB. A greater impact of SDB on nocturnal hypoxaemia than airflow obstruction could be a factor in these findings and survival bias could also have been a confounding factor. A recent study containing data obtained from the National 


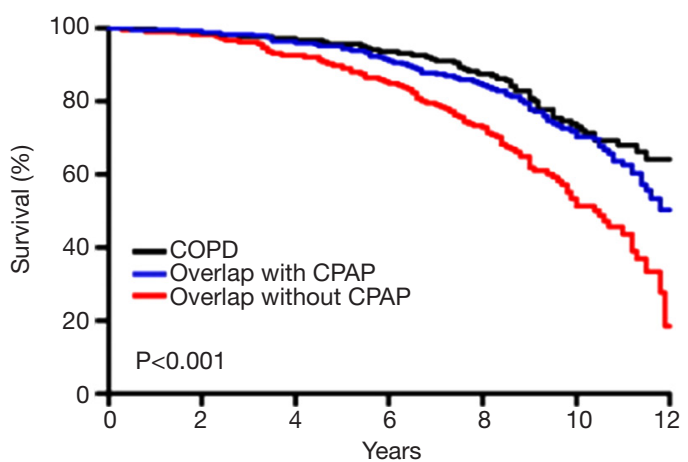

No at risk

$\begin{array}{lrrrrrrr}\text { COPD } & 210 & 203 & 196 & 184 & 144 & 89 & 10 \\ \text { Overlap with CPAP } & 228 & 223 & 215 & 201 & 167 & 97 & 8 \\ \text { Overlap without CPAP } & 213 & 204 & 186 & 161 & 121 & 57 & 3\end{array}$

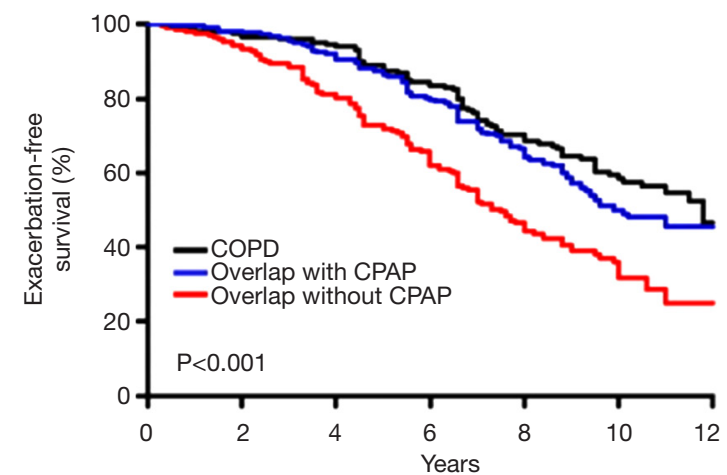

No at risk

$\begin{array}{llllllll}\text { COPD } & 210 & 199 & 189 & 158 & 107 & 47 & 6\end{array}$

$\begin{array}{llllllll}\text { Overlap with CPAP } & 228 & 222 & 202 & 168 & 114 & 41 & 5\end{array}$

$\begin{array}{llllllll}\text { Overlap without CPAP } & 213 & 197 & 165 & 124 & 66 & 24 & 2\end{array}$

Figure 4 Kaplan-Meier survival curves for outcomes among COPD patients without OSA (COPD group), patients with COPD and coexisting OSA (overlap group), and patients with overlap syndrome treated with CPAP since enrolment (overlap with CPAP group). Percentage survival (A), percentage of severe COPD exacerbation-free survival curves among the three study groups (B). The differences between COPD only and COPD with OSA treated with CPAP are statistically significant from patients with COPD and untreated OSA $(\mathrm{P}<0.001)$. COPD, chronic obstructive pulmonary disease; OSA, obstructive sleep apnoea; CPAP, continuous positive airway pressure. Reproduced from (58).

Health and Nutrition Examination Survey (NHANES) reported a trend towards higher mortality in patients with COPD-OSA overlap compared to those with COPD alone (61). Furthermore, Lambert and co-authors recently reported that obesity is associated with increased morbidity in moderate to severe COPD based on data from 3,631 participants from the prospective COPDGene study with spirometry-confirmed COPD (62). While no information is provided regarding co-existing OSA, this possibility could be a contributing factor to the increased morbidity associated with obesity. The increased morbidity principally related to an increased frequency of COPD exacerbations. The likelihood that obesity may have been a marker of undiagnosed OSA is supported by two recent report indicating that high $\mathrm{BMI}$ in COPD is a strong indicator of co-existing OSA $(63,64)$.

\section{Conclusions}

The overlap syndrome is highly prevalent, and should be especially so in the chronic bronchitis COPD phenotype, although specific evidence is lacking. Cardiovascular disease is also prevalent in both COPD and OSA, but there are few published reports on the prevalence of cardiovascular disease in the overlap syndrome other than pulmonary hypertension. Thus, considerable knowledge gaps remain and further research is urgently required, as outlined in a recent research statement by the American Thoracic Society (65). Failure to diagnose overlap syndrome in patients with COPD is likely to result in worse outcomes as CPAP therapy has documented benefits to long term morbidity and mortality in overlap patients, which would not be regarded as standard therapy in COPD alone. Further research is required, especially to document the prevalence of cardiovascular co-morbidity in patients with the overlap syndrome and the basic mechanisms involved, in addition to cardiovascular outcomes of positive pressure therapy.

\section{Acknowledgements}

None.

\section{Footnote}

Conflicts of Interest: The author has no conflicts of interest to declare.

\section{References}

1. Diaz-Guzman E, Mannino DM. Epidemiology and Prevalence of Chronic Obstructive Pulmonary Disease. Clin Chest Med 2014;35:7-16.

2. Senaratna CV, Perret JL, Lodge CJ, et al. Prevalence of obstructive sleep apnea in the general population: A systematic review. Sleep Med Rev 2017;34:70-81. 
3. Heinzer R, Vat S, Marques-Vidal P, et al. Prevalence of sleep-disordered breathing in the general population: the HypnoLaus study. Lancet Respir Med 2015;3:310-8.

4. McSharry DG, Ryan S, Calverley P, et al. Sleep quality in chronic obstructive pulmonary disease. Respirology 2012;17:1119-24.

5. Zeidler MR, Martin JL, Kleerup EC, et al. Sleep disruption as a predictor of quality of life among patients in the subpopulations and intermediate outcome measures in COPD study (SPIROMICS). Sleep 2018;41.

6. McNicholas WT, Bonsignore MR. Sleep apnoea as an independent risk factor for cardiovascular disease: current evidence, basic mechanisms and research priorities. Eur Respir J 2007;29:156-78.

7. Chen W, Thomas J, Sadatsafavi M, et al. Risk of cardiovascular comorbidity in patients with chronic obstructive pulmonary disease: a systematic review and meta-analysis. Lancet Respir Med 2015;3:631-9.

8. McNicholas WT. Chronic Obstructive Pulmonary Disease and Obstructive Sleep Apnea: Overlaps in Pathophysiology, Systemic Inflammation, and Cardiovascular Disease. Am J Respir Crit Care Med 2009;180:692-700.

9. McNicholas WT. COPD-OSA Overlap Syndrome: evolving evidence regarding epidemiology, clinical consequences, and management. Chest 2017;152:1318-26.

10. White LH, Bradley TD. Role of nocturnal rostral fluid shift in the pathogenesis of obstructive and central sleep apnoea. J Physiol 2013;591:1179-93.

11. Teodorescu M, Xie A, Sorkness CA, et al. Effects of Inhaled Fluticasone on Upper Airway during Sleep and Wakefulness in Asthma: A Pilot Study. J Clin Sleep Med 2014;10:183-93.

12. Biselli P, Grossman PR, Kirkness JP, et al. The effect of increased lung volume in chronic obstructive pulmonary disease on upper airway obstruction during sleep. J Appl Physiol 2015;119:266-71.

13. Krachman SL, Tiwari R, Vega ME, et al. Effect of Emphysema Severity on the Apnea-Hypopnea Index in Smokers with Obstructive Sleep Apnea. Ann Am Thorac Soc 2016;13:1129-35.

14. Zhao YY, Blackwell T, Ensrud KE, et al. Sleep Apnea and Obstructive Airway Disease in Older Men: Outcomes of Sleep Disorders in Older Men Study. Sleep 2016;39:1343-51.

15. Mulloy E, McNicholas WT. Theophylline in obstructive sleep apnea. A double-blind evaluation. Chest 1992;101:753-7.

16. Kim V, Han MK, Vance GB, et al. The Chronic Bronchitic
Phenotype of COPD: An Analysis of the COPDGene Study. Chest 2011;140:626-33.

17. Wang Y, Hu K, Liu K, et al. Obstructive sleep apnea exacerbates airway inflammation in patients with chronic obstructive pulmonary disease. Sleep Med 2015;16:1123-30.

18. Tuleta I, Stöckigt F, Juergens UR, et al. Intermittent Hypoxia Contributes to the Lung Damage by Increased Oxidative Stress, Inflammation, and Disbalance in Protease/Antiprotease System. Lung 2016;194:1015-20.

19. Soler X, Gaio E, Powell FL, et al. High Prevalence of Obstructive Sleep Apnea in Patients with Moderate to Severe Chronic Obstructive Pulmonary Disease. Ann Am Thorac Soc 2015;12:1219-25.

20. Steveling EH, Clarenbach CF, Miedinger D, et al. Predictors of the overlap syndrome and its association with comorbidities in patients with chronic obstructive pulmonary disease. Respiration 2014;88:451-7.

21. Sanders $M H$, Newman AB, Haggerty CL, et al. Sleep and Sleep-disordered Breathing in Adults with Predominantly Mild Obstructive Airway Disease. Am J Respir Crit Care Med 2003;167:7-14.

22. Greenberg-Dotan S, Reuveni H, Tal A, et al. Increased prevalence of obstructive lung disease in patients with obstructive sleep apnea. Sleep Breath 2014;18:69-75.

23. Shawon MSR, Perret JL, Senaratna CV, et al. Current evidence on prevalence and clinical outcomes of comorbid obstructive sleep apnea and chronic obstructive pulmonary disease: A systematic review. Sleep Med Rev 2017;32:58-68.

24. Bednarek M, Plywaczewski R, Jonczak L, et al. There is no relationship between chronic obstructive pulmonary disease and obstructive sleep apnea syndrome: a population study. Respiration 2005;72:142-9.

25. Yin HL, Yin SQ, Lin QY, et al. Prevalence of comorbidities in chronic obstructive pulmonary disease patients: A metaanalysis. Medicine 2017;96:e6836.

26. MacDonald MI, Shafuddin E, King PT, et al. Cardiac dysfunction during exacerbations of chronic obstructive pulmonary disease. Lancet Respir Med 2016;4:138-48.

27. Buchan A, Bennett R, Coad A, et al. The role of cardiac biomarkers for predicting left ventricular dysfunction and cardiovascular mortality in acute exacerbations of COPD. Open Heart 2015;2:e000052.

28. Dong JY, Zhang YH, Qin LQ. Obstructive sleep apnea and cardiovascular risk: Meta-analysis of prospective cohort studies. Atherosclerosis 2013;229:489-95.

29. Donato L, Elisiana CG, Giulia P, et al. Prevalence of 
comorbidities in patients with obstructive sleep apnea syndrome, overlap syndrome and obesity hypoventilation syndrome. Clin Respir J 2018;12:1905-11.

30. Chaouat A, Naeije R, Weitzenblum E. Pulmonary hypertension in COPD. Eur Respir J 2008;32:1371-85.

31. Fletcher EC, Luckett RA, Miller T, et al. Pulmonary vascular hemodynamics in chronic lung disease patients with and without oxyhemoglobin desaturation during sleep. Chest 1989;95:757-64.

32. Sharma B, Neilan TG, Kwong RY, et al. Evaluation of Right Ventricular Remodeling Using Cardiac Magnetic Resonance Imaging in Co-Existent Chronic Obstructive Pulmonary Disease and Obstructive Sleep Apnea. COPD 2013;10:4-10.

33. Ganga HV, Nair SU, Puppala VK, et al. Risk of newonset atrial fibrillation in elderly patients with the overlap syndrome: a retrospective cohort study. J Geriatr Cardiol 2013;10:129-34.

34. Castro-Grattoni AL, Alvarez-Buvé R, Torres M, et al. Intermittent Hypoxia-Induced Cardiovascular Remodeling Is Reversed by Normoxia in a Mouse Model of Sleep Apnea. Chest 2016;149:1400-8.

35. Fabbri LM, Luppi F, Beghe B, et al. Complex chronic comorbidities of COPD. Eur Respir J 2008;31:204-12.

36. Turnbull CD, Sen D, Kohler M, et al. Effect of Supplemental Oxygen on Blood Pressure in Obstructive Sleep Apnea (SOX): A Randomised, CPAP Withdrawal Trial. Am J Respir Crit Care Med. [Epub ahead of print].

37. Lavie L. Obstructive sleep apnoea syndrome - an oxidative stress disorder. Sleep Med Rev 2003;7:35-51.

38. McNicholas WT. Chronic obstructive pulmonary disease and obstructive sleep apnoea-the overlap syndrome. J Thorac Dis 2016;8:236-42.

39. Ryan S, Taylor CT, McNicholas WT. Selective Activation of Inflammatory Pathways by Intermittent Hypoxia in Obstructive Sleep Apnea Syndrome. Circulation 2005;112:2660-7.

40. Ryan S, Taylor CT, McNicholas WT. Predictors of elevated nuclear factor-kappaB-dependent genes in obstructive sleep apnea syndrome. Am J Respir Crit Care Med 2006;174:824-30.

41. Rius J, Guma M, Schachtrup C, et al. NF- $\kappa$ B links innate immunity to the hypoxic response through transcriptional regulation of HIF-1 $\alpha$. Nature 2008;453:807.

42. Haarmann H, Folle J, Nguyen XP, et al. Sympathetic Activation is Associated with Exercise Limitation in COPD. COPD 2016;13:589-94.

43. Crinion SJ, Ryan S, McNicholas W'T. Obstructive sleep apnoea as a cause of nocturnal nondipping blood pressure: recent evidence regarding clinical importance and underlying mechanisms. Eur Respir J 2017;49.

44. Ryan S, Crinion SJ, McNicholas WT. Obesity and sleepdisordered breathing-when two 'bad guys' meet. QJM 2014;107:949-54.

45. Agusti A, Morla M, Sauleda J, et al. NF-\{kappa\}B activation and iNOS upregulation in skeletal muscle of patients with COPD and low body weight. Thorax 2004;59:483-7.

46. Taranto-Montemurro L, Messineo L, Perger E, et al. Cardiac Sympathetic Hyperactivity in Patients with Chronic Obstructive Pulmonary Disease and Obstructive Sleep Apnea. COPD 2016;13:706-11.

47. Zangrando KT, Trimer R, de Carvalho LCS, et al. Chronic obstructive pulmonary disease severity and its association with obstructive sleep apnea syndrome: impact on cardiac autonomic modulation and functional capacity. Int J Chron Obstruct Pulmon Dis 2018;13:1343-51.

48. Pizarro C, van Essen F, Linnhoff F, et al. Speckle tracking echocardiography in chronic obstructive pulmonary disease and overlapping obstructive sleep apnea. Int J Chron Obstruct Pulmon Dis 2016;11:1823-34.

49. Grote L, Sommermeyer D, Ficker J, et al. REM Sleep Imposes a Vascular Load in COPD Patients Independent of Sleep Apnea. COPD 2017;14:565-72.

50. McNicholas WT, Calverley PM, Lee A, et al. Long-acting inhaled anticholinergic therapy improves sleeping oxygen saturation in COPD. Eur Respir J 2004;23:825-31.

51. Ryan S, Doherty LS, Rock C, et al. Effects of Salmeterol on Sleeping Oxygen Saturation in Chronic Obstructive Pulmonary Disease. Respiration 2010;79:475-81.

52. Mulloy E, McNicholas WT. Theophylline improves gas exchange during rest, exercise, and sleep in severe chronic obstructive pulmonary disease. Am Rev Respir Dis 1993;148:1030-6.

53. Goldstein RS, Ramcharan V, Bowes G, et al. Effect of supplemental nocturnal oxygen on gas exchange in patients with severe obstructive lung disease. $\mathrm{N}$ Engl J Med 1984;310:425-9.

54. Weitzenblum E, Chaouat A, Kessler R, et al. Overlap syndrome: obstructive sleep apnea in patients with chronic obstructive pulmonary disease. Proc Am Thorac Soc 2008;5:237-41.

55. Struik FM, Lacasse Y, Goldstein R, et al. Nocturnal noninvasive positive pressure ventilation for stable chronic obstructive pulmonary disease. Cochrane Database Syst Rev 2013;CD002878. 
56. Köhnlein T, Windisch W, Köhler D, et al. Noninvasive positive pressure ventilation for the treatment of severe stable chronic obstructive pulmonary disease: a prospective, multicentre, randomised, controlled clinical trial. Lancet Respir Med 2014;2:698-705.

57. Murphy PB, Rehal S, Arbane G, et al. Effect of home noninvasive ventilation with oxygen therapy vs oxygen therapy alone on hospital readmission or death after an acute copd exacerbation: A randomized clinical trial. JAMA 2017;317:2177-86.

58. Marin JM, Soriano JB, Carrizo SJ, et al. Outcomes in Patients with Chronic Obstructive Pulmonary Disease and Obstructive Sleep Apnea. Am J Respir Crit Care Med 2010;182:325-31.

59. Machado MC, Vollmer WM, Togeiro SM, et al. CPAP and survival in moderate-to-severe obstructive sleep apnoea syndrome and hypoxaemic COPD. Eur Respir J 2010;35:132-7.

60. Putcha N, Crainiceanu C, Norato G, et al. Influence of Lung Function and Sleep-disordered Breathing on AllCause Mortality. A Community-based Study. Am J Respir Crit Care Med 2016;194:1007-14.

61. Du W, Liu J, Zhou J, et al. Obstructive sleep apnea,

Cite this article as: McNicholas WT. Comorbid obstructive sleep apnoea and chronic obstructive pulmonary disease and the risk of cardiovascular disease. J Thorac Dis 2018;10(Suppl 34):S4253-S4261. doi: 10.21037/jtd.2018.10.117
COPD, the overlap syndrome, and mortality: results from the 2005-2008 National Health and Nutrition Examination Survey. Int J Chron Obstruct Pulmon Dis 2018;13:665-74.

62. Lambert AA, Putcha N, Drummond MB, et al. Obesity Is Associated With Increased Morbidity in Moderate to Severe COPD. Chest 2017;151:68-77.

63. Gunduz C, Basoglu OK, Tasbakan MS. Prevalence of overlap syndrome in chronic obstructive pulmonary disease patients without sleep apnea symptoms. Clin Respir J 2018;12:105-12.

64. Soler X, Liao SY, Marin JM, et al. Age, gender, neck circumference, and Epworth sleepiness scale do not predict obstructive sleep apnea (OSA) in moderate to severe chronic obstructive pulmonary disease (COPD): The challenge to predict OSA in advanced COPD. PLoS One 2017;12:e0177289.

65. Malhotra A, Schwartz AR, Schneider H, et al. Research Priorities in Pathophysiology for Sleep-disordered Breathing in Patients with Chronic Obstructive Pulmonary Disease. An Official American Thoracic Society Research Statement. Am J Respir Crit Care Med 2018;197:289-99. 